\title{
Le curriculum scolaire au Chili
}

Genèse, mise en œuvre et développement

The school curriculum in Chile

Genesis, implementation and development

El currículum escolar en Chile

Génesis, implementación y desarrollo

\section{Cristián Cox}

Traducteur : Jérôme Quintana

\section{CpenEdition}

\section{Journals}

Édition électronique

URL : https://journals.openedition.org/ries/1047

DOI : $10.4000 /$ ries. 1047

ISSN : 2261-4265

Éditeur

France Education international

Édition imprimée

Date de publication : 1 avril 2011

Pagination : 51-61

ISBN : 978-2854205916

ISSN : 1254-4590

Référence électronique

Cristián Cox, "Le curriculum scolaire au Chili », Revue internationale d'éducation de Sèvres [En ligne], 56 | avril 2011, mis en ligne le 01 avril 2014, consulté le 04 mai 2021. URL : http:// journals.openedition.org/ries/1047 ; DOI : https://doi.org/10.4000/ries.1047 


\section{Le curriculum scolaire au Chili}

Genèse, mise en œuvre et développement*

\section{Cristián Cox}

La question principale autour du curriculum et de sa réforme à l'heure actuelle réside dans le changement net et rapide de la culture à partir de laquelle se définissent ses orientations et ses contenus. Elle réside aussi dans l'incertitude quant à l'avenir, notamment sur le plan des connaissances, des aptitudes et des valeurs requises. Au Chili, comme dans de nombreux pays durant les quinze dernières années, le curriculum scolaire a fait l'objet d'importants changements afin de répondre à l'impact de processus tels que la révolution des technologies de l'information et de la communication ou encore la mondialisation. Ces changements visaient à répondre également à l'impact spécifique de l'histoire politique récente du pays qui, au sortir d'un long régime autoritaire, s'est vu confronté aux défis posés par la croissance économique, l'intégration sociale et le développement démocratique. C'est dans ce cadre que le présent article s'intéresse aux quatre questions suivantes: les concepts et instruments qui forment le curriculum scolaire officiel ; les institutions et les processus qui contrôlent son changement ; les tendances et les influences qui ont orienté son dynamisme ; enfin, les caractéristiques de sa mise en œuvre ainsi que les points de vue des acteurs.

\section{CURRICULUM : USAGE HISTORIQUE ET CONFIGURATION ACTUELLE}

Le terme « curriculum » est couramment utilisé dans le système éducatif chilien depuis la dernière partie du XIX siècle, au moment où les autorités éducatives de l'époque, suivant les modèles allemands, ont mis en place un plan d'études concentrique. Durant plus d'un siècle, le concept prédominant dans la norme et la culture enseignantes voulait que par curriculum, on entende la définition donnée par le ministère de l'Éducation : l'ensemble des objectifs et contenus d'apprentissage organisés par domaines de connaissances et par activités, dans une séquence temporelle donnée et avec des charges horaires définies pour chacune de ses unités ou segments (les disciplines d'hier, les secteurs ou domaines curriculaires d'aujourd'hui). Le curriculum a donc, d'un point de vue historique, consisté en la prescription, obligatoire pour l'ensemble du système

* Article traduit par Jérôme Quintana. 
scolaire, d'un "plan d'études ", à savoir la définition de domaines de connaissances, des horaires correspondants et de leur séquencement sur la totalité du parcours scolaire et d'un "programme d'études ", à savoir les objectifs d'apprentissage, les contenus et orientations didactiques pour l'enseignant, organisés par unités pour chaque année scolaire.

Ce concept a été profondément réformé à l'issue du régime autoritaire qui a gouverné le pays, depuis la crise démocratique et le coup d'état militaire de 1973 jusqu'en 1990. La veille du transfert du pouvoir au premier gouvernement de la reprise démocratique (le 10 mars 1990), le gouvernement militaire a promulgué une loi de l'éducation (Loi organique constitutionnelle de l'enseignement: LOCE). Cette loi, pour ce qui est des questions relatives au curriculum, a d'une part établi une distinction entre "cadre curriculaire » et "plans et programmes d'études ", et d'autre part prévu la décentralisation du contrôle de ces derniers, en instaurant le fait que chaque institution scolaire devait décider si elle souhaitait avoir ses propres plans et programmes d'études ou bien appliquer ceux qui avaient été définis par le ministère. En outre, ces nouvelles dispositions ont donné lieu à la mise en place d'un organisme public indépendant du ministère, le Conseil supérieur de l'éducation, qui s'est vu conférer l'autorité ultime en matière de curriculum dans le système scolaire. Depuis lors, toute initiative visant à modifier le curriculum national doit être élaborée par le ministère de l'Éducation et proposée au Conseil pour approbation. ${ }^{1}$

Le concept de "cadre curriculaire d'objectifs fondamentaux et de contenus minimums obligatoires ", défini par la LOCE, correspond à des définitions à la fois larges dans leur portée et concises dans leur forme. Il s'agit d'objectifs et de contenus annuels, dont la forme est assimilable à des standards (même si le terme estándares n’a jamais été utilisé au Chili en relation au curriculum). Ce cadre revêt également un caractère obligatoire pour l'ensemble du système scolaire, public ou privé. ${ }^{2}$ De leur côté, les "plans et programmes d'études » correspondent aux choix didactiques et aux séquences pédagogiques visant à mettre en œuvre les objectifs et les contenus définis dans le cadre. Cette définition inclut les orientations destinées à l'enseignant concernant les acquis attendus, ainsi que des exemples d'activités avec les élèves et des exemples d'évaluation. Comme il a été dit, les programmes d'études sont optionnels, ce que n'est pas le cadre : chaque établissement scolaire du pays (d'enseignement

\footnotetext{
1. Le Conseil supérieur de l'éducation est un organisme autonome qui, entre autres fonctions, doit approuver ou rejeter les propositions du cadre curriculaire et des plans et programmes d'études élaborés par le ministère de l'Éducation. En 2010, il change d'appellation et devient le Conseil national de l'éducation. Il est désormais composé seulement de représentants du monde éducatif, excluant les représentants du pouvoir judiciaire et des forces armées (Loi 20.129).

2. L'organisation institutionnelle de l'enseignement scolaire au Chili est tripartite: il existe un enseignement public géré au niveau municipal ( $43 \%$ des effectifs, sur un total de 3,4 millions d'élèves), un enseignement de propriété privée subventionné par l'État (49,6\% des effectifs) et un enseignement privé (tant sur le plan des financements que sur celui de la propriété) qui rassemble 6,7 \% des effectifs. Une catégorie spéciale de lycées technico-professionnels, gérés par des entreprises privées, représentait 1,5\% des effectifs en 2009 [www.mineduc.cl].
} 
primaire ou secondaire) doit décider s'il définit son propre programme d'études ou s'il adopte, à l'inverse, les programmes élaborés par le ministère. Dans ce cas, il doit en outre adopter le plan d'études officiel qui définit l'horaire hebdomadaire et annuel établi pour chaque domaine ou discipline du curriculum.

Ces nouvelles définitions sont appliquées depuis maintenant deux décennies et aujourd'hui, moins de $20 \%$ des établissements du pays ont leurs propres plans et programmes d'études. L'immense majorité suit les programmes définis par le ministère de l'Éducation. Dans le premier cas de figure, les établissements doivent s'en tenir aux objectifs et aux contenus définis dans le cadre obligatoire, sous la supervision du ministère.

Cette structure à double niveau - où l'on définit d'une part, de façon centralisée et obligatoire, ce qui doit être fondamentalement inclus dans le curriculum et où, d'autre part, on décentralise les moyens de parvenir aux objectifs - est propre aux réformes curriculaires des années 1990 dans de nombreux pays, tant dans ceux du "premier monde » qu'en Amérique latine. ${ }^{3}$ Mais c'est au Chili que, en ce qui concerne les plans et programmes d'études, on a maintenu une prescription curriculaire officielle, élaborée de manière centralisée.

Aux instruments curriculaires mentionnés, le ministère de l'Éducation a ajouté en 2009 un troisième instrument, dont l'application n'est pas obligatoire, mais qui a été conçu comme soutien. Il s'agit des « cartes de progrès ». Ces cartes décrivent l'évolution en spirale des progrès de l'apprentissage tels qu'on les attend des élèves tout au long des douze années de leur scolarité, par rapport à des axes clés propres aux disciplines choisies dans le curriculum, en distinguant sept niveaux, dont chacun équivaut approximativement à deux années d'études dans le système scolaire. Les cartes de progrès ne définissent pas d'autres apprentissages que ceux définis dans le cadre curriculaire et dans les programmes d'études. Leur valeur réside dans la vue d'ensemble qu'ils proposent sur le plan de la progression de l'apprentissage. ${ }^{4}$

\section{ÉLABORATION : INSTITUTIONS ET PROCESSUS}

Traditionnellement, au Chili les réformes curriculaires ont été élaborées depuis le ministère de l'Éducation avec des équipes ad hoc à la fois internes et externes, dissoutes une fois terminé le processus d'élaboration d'un nouveau plan et programme d'études. Historiquement, les réformes n’ont pas prévu la

\footnotetext{
3. À titre d'exemple, l’Argentine, le Brésil et la Colombie ont défini, dans le dernier tiers des années 1990, des cadres curriculaires appelés estándares (standards).

4. Une façon concrète de visualiser les trois composants de l'architecture curriculaire en question, et d'en appréhender les relations internes, est de considérer leur étendue : le document Cadre traite de la définition des objectifs et des contenus pour une discipline et pour une année d'études, en moyenne en quatre pages ; le document Programme définit les méthodologies, les critères pédagogiques et les exemples d'activités, également pour une discipline et pour une année d'études, en moyenne en 90 pages; chaque document Carte de progrès traite du descriptif d'un niveau d'apprentissage (qui équivaut à deux années d'études) en deux pages.
} 
consultation d'acteurs à l'intérieur ou à l'extérieur du système éducatif. En revanche, le curriculum aujourd'hui en vigueur dans le pays doit sa naissance à une unité spécialisée permanente du ministère, consacrée à la conception, à l'évaluation et au développement du curriculum (Unité du curriculum et de l'évaluation : UCE). Il est également le fruit de consultations larges et systématiques, à l'intérieur du système éducatif (pour les enseignants) comme à l'extérieur (le monde académique universitaire dans le cas de l'enseignement secondaire général, le monde de l'entreprise dans le cas de l'enseignement secondaire technico-professionnel).

Le curriculum en vigueur en 2010 est le résultat d'une réforme plus importante en termes de structure et de contenus, mise en place en 1996 pour l'enseignement de base (de la première à la huitième année d'études) et en 1998 pour l'enseignement secondaire (de la neuvième à la douzième année d'études). Il est également le fruit d'un ajustement des séquences, formats et contenus d'un grand nombre de domaines, ajustement réalisé en 2009 (Ministère de l'Éducation, 2009). Les processus politiques et techniques qui sous-tendent les phases de réforme et d'ajustement dans leur conception et leur définition du curriculum s'inscrivent à la fois dans la continuité et dans la différence.

Le processus d'élaboration de la réforme du curriculum dans les années 1990 comporte deux traits distinctifs qui le singularisent. Il s'agit d'un long processus, qui recouvre la période allant de 1992 à 1995 pour l'enseignement de base et de 1996 à 1998 pour l'enseignement secondaire. Les deux phases voient la participation d'instances importantes hors du champ de l'éducation, qui représentent le monde politique et celui de la production et permettent de les articuler. De même, en ce qui concerne le monde scolaire et le corps enseignant, il n'y a pas eu de processus de consultation systématique sur le curriculum de l'enseignement de base. En revanche, ce fut le cas pour l'enseignement secondaire. On a alors consulté des groupes d'enseignants spécialistes de chaque discipline, ainsi que les établissements du secondaire qui existaient en 1997. Trois instances ont permis cela :

- une Commission nationale pour la modernisation de l'éducation (1994), instance politiquement plurielle et recouvrant un nombre particulièrement important d'institutions, mise en place sur requête du président de la République, qui a élaboré et détaillé un cadre de valeurs pour l'enseignement scolaire et a, par la suite, constitué la base des objectifs transversaux du cadre curriculaire ;

- une consultation nationale (de juin à août 1997) relative à une proposition de curriculum pour l'enseignement secondaire élaborée par le ministère de l'Éducation, qui a concerné un peu plus d'une cinquantaine d'institutions (dont une dizaine d'universités, le Collège des professeurs, la Confédération de la production et du commerce, les forces armées, l'Église catholique, la francmaçonnerie) ainsi que l'ensemble des établissements secondaires du pays ; 
- un long processus (1995-1997) auquel participent divers regroupements corporatifs du domaine de la production qui, en dialogue avec le ministère et les universités, ont élaboré des maquettes de diplôme pour chacune des plus de quarante spécialités formant le nouvel enseignement secondaire technicoprofessionnel (Gysling, 2003).

Un peu plus d'une décennie plus tard, le processus d'ajustement ou d'actualisation du curriculum relève de l'Unité du curriculum et de l'évaluation (UCE) que nous avons mentionnée un peu plus haut. Elle est rattachée au ministère de l'Éducation et gère en parallèle le suivi de la mise en place du curriculum dans les salles de classe. En outre, elle évalue de manière approfondie l'adéquation des objectifs et des contenus sur l'ensemble des douze années de la scolarité, du point de vue du développement des apprentissages chez les élèves. ${ }^{5}$ Dans le processus d'ajustement, diverses consultations ont également joué un rôle décisif : celles du corps enseignant et des établissements scolaires. À la différence du temps de la réforme dans les années 1990, ces consultations n'ont pas été réalisées de façon massive, mais plutôt ciblée, et sur un mode plus récursif et intensif qu'alors. Pour simplifier, on passe de questionnaires massifs à un travail continu d'observation et de discussions avec les équipes d'enseignants et de direction dans les écoles, collèges et lycées, afin de recueillir des preuves du travail des élèves pour l'élaboration des cartes de progrès. On organise également des discussions systématiques dans des groupes cibles autour de la nature et de l'utilisation de ces cartes dans la préparation et l'évaluation des cours.

Le contrôle du curriculum au Chili repose, comme nous l'avons vu, sur deux agences institutionnelles publiques: l'une gouvernementale, l'UCE, rattachée au ministère de l'Éducation; l'autre, de caractère étatique mais non gouvernemental, le Conseil national de l'éducation, formée de représentants des divers champs du système éducatif et de la recherche au Chili. Ce dernier a été créé par les auteurs de la LOCE afin de garantir que le curriculum ne soit uniquement pas le fruit de la volonté d'un gouvernement. Durant les deux décennies où son prédécesseur, le Conseil supérieur de l'éducation, a exercé cette fonction, on peut affirmer que son influence a été positive. Il est en effet intervenu comme "instance de révision" d'initiatives du gouvernement, ce qui a contraint ce dernier à s'engager dans des processus de révision, d'ajustement et de négociation qui ont, de façon générale, rendu la prescription curriculaire nationale plus cohérente et lui ont donné une plus grande légitimité, ainsi qu'une plus grande stabilité.

5. Pour la première fois, on procède à la révision du curriculum à partir d’indicateurs systématiques sur les progrès effectifs réalisés par les élèves dans leur apprentissage, dans chaque discipline. Ce processus essentiel dans l'élaboration des cartes de progrès a été déterminant pour l'ajustement de la séquence des contenus entre les différentes années d'études et entre l'enseignement de base et l'enseignement secondaire. C'est également la première fois que, sur le plan de la conception du curriculum, on n'établit plus de séparation entre les deux niveaux d'enseignement. 
À la suite de cela, on peut remarquer que plus les changements ont été profonds et exigeants, plus grande a été l'ampleur des processus de participation et de consultation. Lorsque les changements ont été des ajustements "à l'intérieur » de structures et de catégories déjà existantes, les processus de consultation et de participation se sont spécialisés et se sont davantage orientés vers l'amélioration des instruments utilisés que vers la construction politique de leur légitimité au sein du corps enseignant.

Dans le même temps, on peut établir un constat de l'analyse des vastes processus de consultation et de participation relatifs à l'innovation en matière de curriculum dans les années 1990. Plus ces processus ont été inclusifs et authentiques, plus ils ont permis de renforcer les bases afin, plus tard, de mettre en place le curriculum, même si les limites de l'innovation se sont également avérées plus grandes. La proposition d'un nouveau curriculum, soumise à la consultation en 1997, était plus novatrice que celle finalement élaborée par le ministère à partir des résultats de cette consultation. Le résultat final peut effectivement se concevoir comme une transaction entre «le changement ordonné de l'extérieur " (par la société à travers le système politique) et "le changement interne à la profession " (provoqué par le système scolaire, à travers le corps enseignant) (Goodson, 2006).

\section{TENDANCES ET INFLUENCES DANS LE DYNAMISME DU CURRICULUM}

Les questions de fond sur ce qui doit constituer le curriculum - quel choix de contenus pour quels apprentissages et sur quels prérequis ces apprentissages doivent-ils être fondés? - ont été abordées à travers les différents processus de réforme et d'ajustement curriculaires au Chili ces quinze dernières années. On a pris en compte les tendances d'ensemble de la société de la connaissance, ainsi que les exigences imposées par l'histoire politique récente du pays, et on a procédé à l'analyse comparative systématique des réformes curriculaires entreprises dans les pays du premier monde et en Amérique latine.

La réponse aux deux questions s'est orientée vers le fait que les tendances séculaires évoquées exigeaient du système scolaire national une plus grande capacité de formation à de nouvelles aptitudes ou, à un niveau plus élevé et de manière plus soutenue, de formation à des aptitudes similaires aux objectifs d'apprentissage traditionnels: capacité d'abstraction, pensée systémique, expérimentation, capacité d'apprendre à apprendre, communication et travail collaboratif, résolution de problèmes, gestion de l'incertitude et adaptation au changement. ${ }^{6}$ L'histoire récente du pays exigeait par ailleurs de mettre l'accent

6. Sur les aptitudes évoquées : vers le milieu et la fin des années 1990, une importante littérature gouvernementale et universitaire y a été consacrée, à l'échelle nationale et internationale. 
sur le développement d'aptitudes et d'attitudes citoyennes fondées sur la valorisation de la démocratie et des droits de l'homme.

Ces deux axes d'exigence sont visibles dans la façon dont le curriculum a intégré le besoin d'apprendre aux jeunes à vivre dans le changement. Comment les enfants et les jeunes peuvent-ils être préparés à cela ? De quels outils ont-ils besoin ? Les fondements du Cadre curriculaire posent le fait que la scolarité doit aider les jeunes à s'orienter dans un monde qui change, et leur donner des bases sûres afin d'atteindre cet objectif. Il n'existe évidemment aucune matière scolaire, sans parler de contenus, dont l'objectif spécifique soit de favoriser une relation adéquate au changement. En revanche, l'ensemble du curriculum tente de fournir les bases de la connaissance et les outils intellectuels propres à une compréhension adéquate de l'histoire contemporaine (fondamentalement grâce au domaine de l'histoire et des sciences sociales) ainsi qu'à une compréhension des relations entre sciences, technologies et société. Le Cadre s'efforce également de fournir une formation morale et relative aux comportements fondée sur des valeurs "d'initiative personnelle, de créativité, de travail en équipe, d'esprit d'entreprise et de relations de confiance mutuelle et responsable " (Objectif fondamental transversal, ministère de l'Éducation, 1998 ; 2009). Il s'agit ensuite de fournir, dans l'ensemble des matières scolaires, les bases et les méthodes nécessaires pour permettre aux élèves d'apprendre à apprendre, de réagir de façon proactive face à l'information, la connaissance et l'apprentissage. Il s'agit enfin d'offrir aussi aux élèves la possibilité de concevoir des points de vue différents des leurs, afin de former des êtres à la fois ouverts, souples, autonomes et doués de sens critique, qui puissent faire leurs propres choix à partir des différentes options qui leur sont offertes. ${ }^{7}$

En ce qui concerne les aptitudes et les attitudes liées à la démocratie et aux droits de l'homme, le changement réside dans le fait que les nouvelles normes éducatives nécessitent de passer de la traditionnelle éducation civique, matière de l'enseignement secondaire qui aborde des connaissances sur la structure et le fonctionnement du gouvernement, à une formation citoyenne, à la fois plus profonde et inclusive. De fait, pour reprendre la littérature académique internationale parue à ce sujet, le changement s'est traduit par le passage d'une conception minimaliste de la formation citoyenne (une seule matière, centrée sur les connaissances, elles-mêmes transmises de façon didactique) à une conception maximaliste (répartie sur diverses matières, centrée tant sur les connaissances que sur les aptitudes, elles-mêmes transmises de façon participative (Kerr, 2002).

En termes de contemporanéité avec les pratiques internationales les plus largement acceptées, il y a eu tout au long du processus d'élaboration curriculaire de la fin des années 1990 et de la fin des années 2000, un élément

7. Le curriculum propose systématiquement divers points de vue pour un même sujet ou une même question, tant en histoire et sciences sociales qu'en sciences. 
important et systématiquement présent: l'analyse curriculaire internationale des expériences récentes et considérées comme particulièrement pertinentes. Sur ce plan, le Chili a dans les années 1990 profité des analyses curriculaires généralisées de la Communauté européenne (European Commission, 1996) et de l'Organisation de coopération et de développement économiques (OCDE), et des documents curriculaires récents liés aux réformes curriculaires mises en place dans les systèmes éducatifs anglais, néo-zélandais, espagnol et argentin, ainsi que dans quelques-uns des États des États-Unis. De la même façon, le Chili a profité de l'analyse curriculaire de la Third International Mathematics and Science Study (TIMSS) et des référentiels nationaux pour l'enseignement des sciences de l'Académie nationale des sciences des États-Unis, afin de définir et d'ajuster ses propres critères dans le domaine des mathématiques et des sciences.

Dans les processus d'ajustement curriculaires conclus en 2009 et 2010, les référents internationaux ont apporté un éclairage plus spécialisé, et leur influence a été probablement plus profonde. Parmi ceux dont l'influence s'est avérée décisive, on mentionnera la célèbre étude de l'OCDE sur la définition et le choix des compétences nécessaires pour le XXI siècle, Definition and Selection of Competencies (DeSeCo), (Rychen et Salganik, 2003). On mentionnera également le cadre conceptuel que représentent les tests du PISA (auxquels le Chili a participé en 2000, 2006 et 2009). On évoquera enfin le travail réalisé sur les cartes de progrès par le Conseil australien pour la recherche en éducation (Australian Council for Research in Education, ACER), institution qui aura joué un rôle décisif dans l'élaboration du cadre conceptuel du PISA.

La problématique autour de l'isomorphisme croissant des systèmes éducatifs (Meyer, Ramírez, 2000) concerne tout autant le Chili que le nombre croissant de pays qui participent aux mesures effectuées par le PISA (soixante-cinq en 2009). Les implications qui découlent de ce fait posent, de façon à la fois nouvelle et intense, la question de savoir quelle peut être la relation la plus féconde et adéquate de chaque système national, ouvert sur le monde mais gardant sa propre identité, avec des critères de plus en plus universels en termes de compétences que les curricula doivent s'efforcer de promouvoir et que les tests internationaux mesurent.

\section{Politiques de mise en GUVRe ET POINT DE VUE DES PROTAGONISTES}

Deux facteurs distincts mais néanmoins cruciaux sont à prendre en compte dans la question de la mise en œuvre du curriculum au Chili : d'une part, la permanence de sa structure, de ses objectifs et de ses contenus pendant plus d'une décennie; d'autre part, son lien structurel étroit avec les systèmes d'évaluation de l'apprentissage des élèves. Cette stabilité permet que, d'année en année, ce qui au départ avait constitué une innovation et exigé beaucoup 
d'efforts de la part du corps enseignant entre 1997 et 2002 (années d'entrée en vigueur du nouveau curriculum dans les différentes années d'études de l'enseignement de base et secondaire), relève en partie de routines connues et, de ce fait, soit réalisé de façon plus complète. L'association avec l'évaluation a par ailleurs joué un rôle décisif. L'éducation au Chili repose sur un système d'évaluation systématique des résultats de l'apprentissage depuis 1988. De plus en plus sophistiqué, depuis la fin des années 1990 le système SIMCE$^{8}$ évalue les résultats en langue, mathématiques, sciences, histoire et sciences sociales, dans trois années d'études de la scolarité : la $4^{\mathrm{e}}$ et la $8^{\mathrm{e}}$ année de l'enseignement de base, et la $2^{\mathrm{e}}$ année de l'enseignement secondaire. Ces tests sont strictement alignés sur le curriculum : de fait, ils ont légalement pour but d'évaluer «la réussite du curriculum $»{ }^{9}$

D'autres composantes des politiques mises en place ont été associées à la mise en œuvre du curriculum dans les classes : le temps scolaire et l'examen d'entrée à l'université. L'allongement de la journée scolaire et la modification de l'examen national d'entrée à l'université sont directement liés à la mise en ouvre du curriculum. Dans les deux cas, il s'est agi de mettre en place des politiques visant à créer les conditions nécessaires de sa mise en œuvre : un "refuge» temporaire élargi pour permettre la mise en œuvre de certains contenus curriculaires plus riches et exigeants. Par ailleurs, l'idée que l'examen le plus important, pour pratiquement tous les élèves qui terminent l'enseignement secondaire, se fonde sur le curriculum, plutôt que sur des " compétences scolaires » (à savoir l'intelligence verbale et mathématique), a contribué de manière décisive à ce que celui-ci fasse l'objet de toutes les attentions de la part des maîtres et des élèves de l'enseignement secondaire.

Parallèlement à ces associations structurelles visant à favoriser la mise en œuvre du curriculum, les politiques de formation continue mises en place pour les enseignants se sont alignées sur le changement curriculaire. Elles ont tout d'abord donné lieu à un nombre important de stages de familiarisation aux nouvelles définitions (dans la période allant de 1997 à 2002), puis à un renforcement des actions de soutien, tout en en diminuant leur étendue à travers un large éventail de stratégies (Sotomayor, Walker, 2009).

Du côté des enseignants, les critiques les plus constantes à l'égard du curriculum en vigueur ont eu trait à la quantité excessive de contenus et au fait que, malgré les processus de participation évoqués plus haut, les enseignants ont eu le sentiment "de ne pas avoir été consultés». Certains domaines du curriculum ont fait l'objet de nombreuses critiques (modifications de la séquence en histoire, excès de contenus en biologie, etc.) auxquels les processus d'ajustement postérieurs ont permis de répondre.

8. SIMCE : Système de mesure de la qualité de l'éducation mis en place par le ministère chilien de l'Éducation (NdT). 9. Voir aussi Cariola et Meckes (2008). "L'évolution du rôle du système d'évaluation. Le cas du Chili ». Revue internationale d'éducation Sèvres $\mathrm{n}^{\circ} 48$, septembre, p. 37-51. (NdIR). 
La corporation des enseignants a émis une critique globale de nature idéologique, sans retombées pratiques : "le curriculum promu dans l'actuel système éducatif est une atteinte à la conception de l'être humain et de la société à laquelle nous aspirons. " Dans le même texte, néanmoins, l'organisation corporative reconnait dans la prescription curriculaire nationale la valeur des " Objectifs fondamentaux transversaux qui tendent à favoriser une formation complète et éthique» (Colegio de Profesores, 2005 : 26-27).

En mai 2006, les lycéens du pays, avec l'appui de la société et des médias, ont occupé les lycées et collèges pour protester contre la mauvaise qualité de l'enseignement, ouvrant ainsi une ère de remise en cause et d'élaboration politico-légale de nouvelles bases institutionnelles du système éducatif du pays. Les protestations n'avaient toutefois pas trait aux objectifs et aux contenus du curriculum. Celui-ci n'a d'ailleurs pas fait l'objet par la suite d'initiatives politico-légales qui remettent en cause son architecture, ses orientations, ni même ses contenus fondamentaux.

Le problème central de la mise en ouvre du curriculum, dans le cas du Chili, n'est pas lié aux critiques du corps enseignant à son encontre. Il ne provient pas non plus d'un manque éventuel de précision des prescriptions, ni même d'un manque de cohérence dans la pression exercée par les politiques mises en œuvre pour aboutir au changement. Il provient en réalité du niveau moyen des compétences des enseignants (OCDE, 2004). Les enseignants du pays n'en sont pas responsables, mais ils possèdent une préparation et une expérience qui correspondent à une étape antérieure du développement de l'éducation dans le pays, à une époque où les exigences de la société vis-à-vis du système scolaire étaient fondées davantage sur l'accès à l'enseignement que sur la qualité de celui-ci. Ainsi, c'est au cour de la problématique actuelle de la mise en œuvre du curriculum au Chili que s'opèrent la sélection et la formation des enseignants.

\section{BibLIOgRAPHIE}

Colegio de Profesores (2006) : "Conclusiones Finales Congreso Pedagógico Curricular 2005 », Revista Docencia, n 28.

GOODSON I. (2006): "Socio-historical processes of curricular change", in A. Benavot, C. Braslavsky (ed.), School Knowledge in Comparative and Historical Perspective. Hong Kong: Springer.

GYSLING J. (2003) : "La reforma curricular: itinerario de una transformación cultural », in C. Cox (ed.), Políticas educacionales en el cambio de siglo, Editorial Universitaria, Santiago. 
European Commission (1996) : Teaching and Learning - Towards the Learning Society. White Paper on Education and Training, Luxembourg.

KERR D. (2003) : "An international review of citizenship in the curriculum: the IEA national case studies and the Inca archive ", in G. Steiner-Khamsi, J. Torney-Purta and J. Schwille, (ed.) New paradigms and recurring paradoxes in education for citizenship: an international comparison, JAI, Elsevier Science, Amsterdam.

LUGINBÜHL O. (1996) : "Curriculum et programmes", Revue internationale d'éducation Sèvres, $\mathrm{n}^{\circ} 12$, décembre.

MEYER J.W. and RAMÍREZ F.O. (2000) : «The world institutionalization of education ", in Discourse Formation in Comparative Education, J. Schriewer, Frankfurt: Peter Lang Publishers.

Ministerio de Educación (2009): Marco de Objetivos Fundamentales y Contenidos Minimos Obligatorios de Educación Básica y Media. Actualización 2009. Santiago.

OCDE (1994): The Curriculum Redefined: Schooling for the 21st century, Paris.

OCDE (2004): Examens des politiques nationales d'éducation. Chili. Paris.

RYCHEN D.S., SALGANIK L.H. (ed.) (2003) : Key competencies for a successful life and a well functioning society. Hogrefe \& Huber, Gottingen.

SOTOMAYOR C. et WALKER H. (editors) (2008) : Formación continua de profesores. Santiago: Editorial Universitaria. 
